\title{
Las diferentes concepciones de la universidad digital en Iberoamérica
}

\section{Different conceptions of digital university in Ibero- America}

\author{
Jesús Salinas Ibáñez \\ Universitat de les Illes Balears (España) \\ Victoria I. Marín Juarros \\ Carl von Ossietzy Universität Oldenburg (Alemania)
}

\section{Resumen}

En los últimos tiempos, se ha oído en muchas ocasiones hablar de la universidad digital en diversos contextos. Sin embargo, no hay una definición clara del concepto que ayude a concretar más su implementación. Por ello, este estudio se ha centrado en analizar las diferentes concepciones existentes de la universidad digital en Iberoamérica, mediante un análisis del campo semántico a través de análisis de redes y una revisión sistemática de literatura para identificar qué entienden las diferentes universidades iberoamericanas por ser una universidad digital. Se utilizaron palabras clave para la búsqueda partiendo de los tesauros e incluyendo la de "universidad digital" a través de diversos catálogos y bases de datos, y se localizaron documentos de diferentes tipos entre 2007 y 2017. La revisión de la literatura, a partir de los resúmenes de los trabajos, contempló la creación de categorías con diversos temas que representan cómo entiende Iberoamérica la universidad digital y con qué restricciones se topa. La discusión incorpora la integración de dichas categorías y temas a los modelos de universidad digital identificados previamente. A modo de conclusión, se presenta una síntesis sobre el concepto de universidad digital y apuntamos algunas notas hacia la realización de dicha concepción.

Palabras clave: universidad a distancia; investigación sobre literatura científica; análisis conceptual; análisis de redes; América Latina; España.

\begin{abstract}
Recently, the term of digital university has been a buzzword or trend in different contexts in Spanish-speaking countries. However, there is no available clear definition of the concept that helps us specify its implementation further. Therefore, this study was aimed at analyzing the different existing conceptions of digital university in Ibero-America, via a systematic literature review and the analysis of the semantic field through a network analysis. This enabled us to identify what was understood by being a digital university from the perspective of different Ibero-American universities. Spanish keywords derived from the thesaurus -including "digital
\end{abstract}


university"- were used in the search through diverse catalogs and databases, and documents of different kinds were found between 2007 and 2017. The literature review was based on the abstracts of the documents and we considered the creation of categories with diverse topics that represent how Ibero-America understands the digital university and with which restrictions has to deal with. The discussion incorporates the integration of those categories and topics into the digital university models that were previously identified. As a conclusion, a summary on the concept of digital university is presented and we point towards some remarks so that the conception is made a reality in its practice.

Keywords: open university; research of academic literature; concept analysis; network analysis; Latin America; Spain.

Las universidades se encuentran en un momento crítico para la transformación desde su formato tradicional hacia otros formatos futuros imprecisos, pero tendrán que responder a tensiones producidas por la evolución de un mundo digital por la presencia de nuevas generaciones con sus características y por la globalización en todos los terrenos. Para denominar a la nueva universidad que responda a estas tensiones utilizamos el concepto de 'universidad digital', que es un concepto que nadie se atreve a definir claramente, pero que aparece de forma explícita o implícita en los trabajos de prospectiva sobre la universidad.

Para encuadrar el tema con algo de perspectiva, quizá sea adecuado hacer referencia a los informes que la CRUE ha ido realizando anualmente a partir de 2006, los conocidos como Informes UNIVERSITIC que, en 2013, publican una edición Latinoaméricana (Fernández y Llorens, 2013).

Los indicadores utilizados han ido variando con los años, pero nos pueden dar una idea del proceso de digitalización de las universidades y, sobre todo, de los elementos considerados en dichos procesos. Dichos indicadores son agrupados en dos niveles (Uceda, 2011; Piriz, 2013):

- Indicadores de descripción de las Tecnologías de la Información (TI), que permiten conocer las TI implantadas en las universidades desde cinco ejes diferentes: enseñanza/aprendizaje; investigación; procesos de gestión; gestión de la información; formación y cultura TI.

- Indicadores de gestión de las TI, que sirven para analizar cuáles son las buenas prácticas en gestión de las TI en las universidades, desde el punto de vista de recursos TI, proyectos TI, servicios TI, dirección de las TI, calidad, normativa y estándares TI y colaboración.

Por su parte, la RedCLARA propulsó a partir de 2011 la Red Directores de Tecnologías de la Información y Comunicación (TIC) de las universidades de América Latina que realiza un encuentro anual (TICAL) en el que se comparten experiencias, 
información y buenas prácticas. Las Actas de los sucesivos encuentros constituyen también una fuente de información sobre la situación de las universidades en el terreno digital.

La perspectiva, desde la que aquí queremos trabajar, se orienta a la enseñanza y la investigación, pero es obvio que estos aspectos no pueden separarse de otras funciones de las tecnologías digitales en la universidad y cuyo impacto influye en la vinculación con otras instituciones y con la sociedad, en cómo afrontar la globalización, en la gestión de la propia institución, en la transformación de los canales de comunicación y de acceso al conocimiento.

En este trabajo, proponemos una revisión sistemática de literatura en torno al concepto de "universidad digital" de acuerdo con sus múltiples concepciones, descritas en el siguiente apartado. Dadas las circunstancias que concurren en la temática, objeto de este trabajo, las características de la revista RIED y del monográfico en que el mismo se incluye, delimitamos el ámbito de estudio al Espacio Común de Educación Superior ALCUE, al abarcar América Latina, Europa y el Caribe. Por otra parte, la revisión la hacemos preferentemente sobre trabajos publicados en castellano, si bien se consideran también trabajos publicados en inglés siempre y cuando los contenidos se refieran al ámbito del estudio delimitado.

\section{CONCEPCIONES DE UNIVERSIDAD DIGITAL}

En una primera revisión de los tesauros disponibles en el campo educativo, se constata que el término "universidad digital" no aparece, probablemente, dada su actualidad. Por otro lado, tampoco se localiza ningún tipo de definición formal.

Quizá lo más cercano a una definición es el trabajo del modelo de universidad digital (Laviña y Mengual, 2008), que llega a pormenorizar los elementos que la conformarían. García-Peñalvo (2011) reproduce y despliega este mismo modelo, pero sin una definición formal.

Por otra parte, una búsqueda con el motor genérico de Google arroja por encima de 82.000 referencias, pero la mayor parte son, sobre todo, propuestas de unidades académicas o institucionales y en casi ningún caso describen experiencias, sino que podrían catalogarse como publicidad institucional para captar estudiantes.

Para entender bien el concepto, quizá convenga comenzar con el término "digitalización" aplicado al mundo universitario, que nos ayudará a acotar el de "universidad digital". Leiva-Aguilera (2016), se refiere a la integración de las herramientas tecnológicas y los recursos digitales desde varios puntos de vista, y pone como ejemplos: "gestión administrativa y académica; modalidad de oferta académica (de presencial a semipresencial u online); difusión de la oferta y actividades; actividades de investigación; e integración de herramientas y recursos digitales en el proceso de aprendizaje" (Leiva-Aguilera, 2016, p. 79).

Este autor señala como una buena forma de acercarse al fenómeno referirse al concepto de "transformación digital", dado que se refiere a la integración de la 
tecnología en el día a día de la organización para mejorarla. Estaríamos hablando de la transformación digital en las universidades, y en la perspectiva de este trabajo, de la integración de herramientas y recursos digitales en los procesos de aprendizaje para mejorar la docencia y la investigación en la educación superior. En esta línea de la transformación digital de las organizaciones educativas, se maneja el Marco Europeo para Organizaciones Educativas Digitalmente Competentes (Kampylis, Punie y Devine, 2015).

En el meta-análisis de Siemens, Gasevic y Dawson (2015), se sitúa como elemento central de la universidad digital el paso de los procesos de aprendizaje en entornos físicos al aprendizaje digital, apuntando a distintas áreas que necesitan atención en la investigación de este asunto: desarrollo docente, cambio organizacional, prácticas innovadoras y nuevos modelos institucionales, efectividad de las actividades de enseñanza y aprendizaje, experiencia estudiantil, éxito creciente para todos los estudiantes y políticas, estrategias y modelos de financiamiento estatales y provinciales.

En una línea parecida, Jones (2013) apunta que la universidad digital es un término que necesita un mayor desarrollo y elaboración. Para este autor, el término "universidad digital" pretende diferenciar las universidades respaldadas por las TIC y las universidades anteriores apoyadas en tecnologías analógicas, y apunta a tres temas clave: el perfil cambiante del estudiante, la idea de alfabetización digital y el aprendizaje digital y las nociones de una nueva universidad invisible.

Derivado de una primera exploración no sistemática de documentación vinculada al concepto, se localizan diversos significados:

a. Enfoque estratégico / Unidad académica (parte o universidad completa).

Se refiere a unidades académicas o instituciones completas que gestionan o se dedican a la educación a distancia utilizando TIC. Se podría asimilar entonces a universidad virtual. Si analizamos los primeros hallazgos en esta búsqueda, encontramos que hace referencia a "implantar y gestionar estos servicios digitales en los diferentes ámbitos que abarca la universidad", "un sistema de secretaría digital para la gestión de los procesos administrativos de la universidad"2, "hacer universidad a través de plataformas digitales, con el fin de acercar la tradición y calidad de nuestra casa de estudios a la comunidad regional y nacional" 3 y, en la mayoría de casos, se trata de ofertas de cursos a distancia en entornos virtuales.

b. Práctica: cultura digital en la universidad.

Otro de los significados hace referencia a las prácticas dentro del proceso de enseñanza, pero también en otros ámbitos (bibliotecas, investigación, comunicación). Freire y Schuch (2010) hacen referencia a ello: "La educación, como proceso basado en conocimiento, comunicación e interacciones sociales se ha visto afectada de forma 
radical por la emergencia de la cultura digital que ha transformado a sus actores, profesores y estudiantes" (p.89) y, para ello, reclaman nuevos modelos pedagógicos. En esta misma línea, Lara (2009) señala que

la Universidad tiene la responsabilidad de definir su identidad digital a partir de las decisiones que tome con respecto a los procesos de identidad de sus miembros, su relación con el conocimiento abierto y su capacidad para flexibilizar sus estructuras internas. En este proceso de reconfiguración dentro de la cultura digital, la Universidad no se concibe como el lugar donde se accede al conocimiento - al menos no en el sentido clásico y tampoco de forma exclusiva-, sino como un espacio de experiencia de aprendizaje y construcción colaborativa del mismo (p. 19).

c. Tendencia o desafíos.

Es frecuente que universidad digital, digitalización o transformación digital aparezca en distintos informes a lo largo de estos últimos años. Los distintos informes UNIVERSITIC de la CRUE mencionados se hacen eco desde el primer momento (Fernández y Llorens, 2013; Gómez, 2016; Uceda, 2011; Piriz, 2013). Pero también en otros ámbitos, como en Musse (2015), para quien la era digital, la globalización y la irrupción de las nuevas generaciones de alumnos, son los ejes principales de la problemática y desafíos planteados a la universidad del siglo XXI.

En TICAL (2016), en una línea similar, se menciona que

la tecnología de la información es estratégica para potenciar la excelencia, expansión, internacionalización e inclusión en las universidades; la educación es el agente transformador para nuestra región, y las TIC tienen la obligación de responder de forma innovadora y eficiente a estas necesidades (p. 12).

\section{d. Presencia en redes.}

En relación a la presencia de las universidades en las redes, Price (2017) señala: "Es claro entonces que las redes sociales juegan un rol muy relevante en la estrategia de comunicación de las IES, tanto con su comunidad (estudiantes, profesores, personal), como con futuros estudiantes y con la comunidad en general" (p. 70).

Otro aspecto clave es la difusión y transferencia de los resultados de investigación. Esto ha llevado a TICAL 2016, p.e., a abrir un eje temático exclusivo para recibir las experiencias de las TIC como apoyo a la investigación. La difusión de los resultados de las investigaciones científicas es fundamental para el desarrollo de la ciencia y ello depende, en gran medida, del posicionamiento de las universidades en la red (Aguado-López, Rogel-Salazar, Becerril-García y Baca-Zapata, 2009).

Esta preocupación ha dado pie en Europa a la iniciativa conjunta de EUNIS y euroCRIS, de recoger información sobre las soluciones tecnológicas que apoyan la 
investigación y analizar sus vínculos con otros sistemas utilizados en las instituciones de educación superior (Ribeiro, de Castro y Minelli, 2016).

A partir de esta experiencia y motivados por los objetivos del Proyecto BELLA-T (Construyendo el enlace de Europa con América Latina), se organiza, de forma conjunta, con TICAL 2017 el Primer Encuentro Latinoamericano de e-Ciencia, generándose un nuevo espacio para el intercambio de conocimientos y buenas prácticas en el uso de las TIC en las labores de investigación, con vistas a contribuir a la mejora y optimización de la gestión y quehacer de los grupos científicos de la América Latina (Larenas, 2017, p. 12).

\section{METODOLOGÍA}

Para explorar la pregunta de investigación "¿Cuáles son las diferentes concepciones de universidad digital en Iberoamérica?”, se llevó a cabo una revisión sistemática de literatura con una previa fase de análisis del campo semántico, como se describe en las fases siguientes:

1. Fase preparatoria. Se realizaron dos actividades:

1.1. Definición de palabras clave, bases de datos, tipo de documentos y período para la búsqueda.

Nos dirigimos a los tesauros disponibles en el campo educativo, y aunque no aparece el término "universidad digital", sí se reconocen otros términos afines que se van a poder contemplar en la revisión de literatura. En la tabla 1 se incluye el listado de tesauros consultados y los términos relacionados con "universidad digital" a utilizar en la búsqueda que se realizará posteriormente:

Tabla 1. Relación de tesauros y términos afines al concepto de "universidad digital"

\begin{tabular}{|l|l|}
\hline \multicolumn{1}{|c|}{ Tesauro } & \multicolumn{1}{c|}{ Términos afines a "universidad } \\
& \multicolumn{1}{c|}{ digital" } \\
\hline Tesauro de la UNESCO & $\begin{array}{l}\text { Universidad abierta } \\
\text { Universidad on line } \\
\text { Universidad a distancia } \\
\text { Universidad sin barreras } \\
\text { Universidad virtual } \\
\text { Teleuniversidad }\end{array}$ \\
\hline $\begin{array}{l}\text { EuroVox Multilingual Thesaurus of the } \\
\text { European Union }\end{array}$ & $\begin{array}{l}\text { Universidad a distancia } \\
\text { Universidad abierta }\end{array}$ \\
\hline $\begin{array}{l}\text { Tesauro Europeo de los Sistemas Educativos } \\
\text { (TESE) de EURYDICE }\end{array}$ & Universidad a distancia \\
\hline
\end{tabular}


Las palabras clave utilizadas incluyen: "universidad digital", "universidad a distancia", "universidad abierta", "universidad on line", "universidad sin barreras", "universidad virtual" y "teleuniversidad". Se valora la posibilidad de incorporar otras palabras clave a partir de la revisión de trabajos con estas, que usen otras similares que puedan ayudar a identificar documentación de interés para el estudio.

Respecto a las bases de datos empleadas, se contemplan aquellas que son reconocidas por su relevancia en el ámbito iberoamericano: Dialnet; Redalyc - Red de Revistas Científicas de América Latina y el Caribe, España y Portugal; Scielo; Latindex, y REDIB: Red Iberoamericana de Innovación y Conocimiento Científico. Se valora, asimismo, la posibilidad de incorporar otras durante la búsqueda si la cantidad de resultados obtenidos se considera insuficiente.

Por la actualidad del tema, se optó por incluir en la búsqueda y revisión artículos científicos con y sin revisión de pares, tesis doctorales, libros y capítulos de libro, informes y actas de congresos, publicados en los últimos 10 años (2007-2017), si bien también se revisaron inicial y superficialmente trabajos previos.

1.2. Tabulación de resultados en tabla Excel incluyendo bases de datos consultadas, título del trabajo, autores, palabras clave, resumen y fecha de publicación. La selección de documentos de relevancia se basó en el título, palabras clave y en el resumen que proporcionan las bases de datos.

2. Segundafase. Análisis del campo semántico a partir de la búsqueda y utilizando las palabras claves emergentes en la búsqueda y selección de los diferentes documentos de relevancia para entender las concepciones de universidad digital, siguiendo el método de análisis de redes.

3. Tercera fase. Revisión sistemática de la literatura mediante la agrupación de categorías y subtemas.

A continuación, se describen con mayor detalle la segunda y tercera fase del proceso llevado a cabo y los resultados hallados.

\section{Análisis del campo semántico}

Como se ha mencionado, el concepto de universidad digital es frecuentemente utilizado, pero su definición sigue siendo muy ambigua, encontrando diferentes interpretaciones del mismo en la literatura sobre educación. Universidad digital, en otras palabras, presenta múltiples significados discursivos que se están considerando como si cada uno fuera el único significado académico posible.

Así, como se ha indicado previamente, en la búsqueda se contempló la posibilidad de incluir otras palabras y se incorporó 'universidad extendida' por su presencia en 
múltiples trabajos como sinónimo próximo al de universidad virtual, y se eliminó 'universidad sin barreras', por no arrojar ningún resultado relevante. Asimismo, tras la búsqueda en las bases de datos inicialmente definidas, se valoró también el uso del buscador Google Académico de modo complementario para poder ampliar resultados.

En la búsqueda se identificaron a fecha de noviembre de 2017, 112 documentos (ver tabla 2), de entre los cuales se encontraban mencionados 132 descriptores. De ellos, se eliminaron los nombres propios de revistas, universidades, etc. y se agruparon sinónimos y conceptos que hacían referencia a campos semánticos similares. Así, se redujo la cantidad a 78 palabras clave que pueden identificar los significados más influyentes para la definición de universidad digital.

Tabla 2. Relación del número de documentos obtenidos a través de la búsqueda por año y palabra clave

\begin{tabular}{|c|c|c|c|c|c|c|c|c|}
\hline \multirow[b]{2}{*}{ Año } & \multirow[b]{2}{*}{$\begin{array}{l}\text { Número de } \\
\text { documentos }\end{array}$} & \multicolumn{7}{|c|}{ Palabras clave ( $n^{0}$ doc.) } \\
\hline & & $\begin{array}{l}\text { Univ. } \\
\text { digital }\end{array}$ & $\begin{array}{c}\text { Univ. a } \\
\text { distancia }\end{array}$ & $\begin{array}{l}\text { Univ. } \\
\text { online }\end{array}$ & Teleuniversidad & $\begin{array}{c}\text { Univ. } \\
\text { Extendida }\end{array}$ & $\begin{array}{l}\text { Univ. } \\
\text { virtual }\end{array}$ & $\begin{array}{l}\text { Univ. } \\
\text { abierta }\end{array}$ \\
\hline 2007 & 5 & 1 & $\mathrm{O}$ & $\mathrm{O}$ & 0 & 1 & 1 & 2 \\
\hline 2008 & 16 & 4 & 2 & 2 & 1 & 4 & 3 & o \\
\hline 2009 & 7 & 4 & 2 & $\mathrm{O}$ & o & $\mathrm{O}$ & 1 & $\mathrm{O}$ \\
\hline 2010 & 16 & 3 & 3 & $\mathrm{O}$ & o & $\mathrm{O}$ & 5 & 5 \\
\hline 2011 & 10 & 5 & 1 & 1 & 1 & $\mathrm{O}$ & 1 & 1 \\
\hline 2012 & 4 & 1 & 1 & $\mathrm{O}$ & o & $\mathrm{O}$ & 1 & 1 \\
\hline 2013 & $\mathbf{1 1}$ & 1 & 2 & 1 & 1 & $\mathrm{O}$ & 4 & 2 \\
\hline 2014 & 15 & 5 & o & 1 & 0 & O & 3 & 6 \\
\hline 2015 & 10 & 3 & 3 & 2 & 0 & O & $\mathrm{O}$ & 2 \\
\hline 2016 & 13 & 2 & 5 & 1 & O & $\mathrm{O}$ & 3 & 2 \\
\hline 2017 & 5 & 2 & 1 & $\mathrm{O}$ & 0 & $\mathrm{O}$ & $\mathrm{O}$ & 2 \\
\hline Total & 112 & 31 & 20 & 8 & 3 & 5 & 22 & 23 \\
\hline
\end{tabular}

En una primera instancia, se listaron las conexiones entre las palabras clave de la búsqueda (definidas en la primera fase de la metodología) y los descriptores de los trabajos (ubicados bien en el epígrafe palabras clave, bien en el título), obteniendo 310 conexiones. A éstas se añadieron las conexiones de los descriptores de cada uno de los artículos entre sí. De esta manera se pretendía conocer, por una parte, la frecuencia de aparición de los distintos conceptos y, con ello, su peso en el campo semántico y, por la otra, la cercanía entre ellos obteniendo, en total, 702 conexiones.

Para conseguir que estas conexiones entre los conceptos y las relaciones entre ellas fueran más fáciles de interpretar, se construyó un mapa de la red relacional (Burman, Green y Shanker, 2015; Green, Feinerer y Burman, 2015). A partir de las 
702 relaciones definidas, se construyó con el paquete de visualización de código abierto Gephi (Bastian, Heymann y Jacomy, 2009) una red de conexiones cruzadas que representa los conceptos asociados a universidad digital.

Así, se importaron las palabras clave de búsqueda como nodos y las conexiones como aristas, dando a los nodos un tamaño proporcional a su grado (número de conexiones) y realizando un análisis de la distribución de las 78 palabras clave por su cercanía y número de conexiones unas respecto de otras. Esto proporcionó una nube relacional no filtrada, es decir, que incluye algunas relaciones entre palabras clave que son irrelevantes para nuestro objetivo (identificación de los descriptores y conexiones más importantes para describir el concepto de universidad digital) en aras de trabajar con datos sin procesar previamente.

El grafo resultante (figura 1) describe el peso y relación entre los diferentes conceptos, pudiéndose identificar dos conceptos dominantes -'universidad digital' y educación a distancia'- y una serie de conceptos que destacan en cuanto a su peso como son 'educación superior', 'universidad abierta', 'TIC', 'universidad virtual', 'aprendizaje virtual', 'investigación', 'acceso abierto' y, en un segundo nivel, 'campus virtual', 'universidad extendida', 'ambiente virtual', 'enseñanza virtual', 'innovación educativa', 'recursos educativos abiertos', 'conocimiento abierto', 'política de educación superior', 'trabajo colaborativo' y 'gestión educativa'. 
Figura 1. Mapa de red entre palabras clave de búsqueda y de identificación de trabajos relacionados en torno al concepto de universidad digital

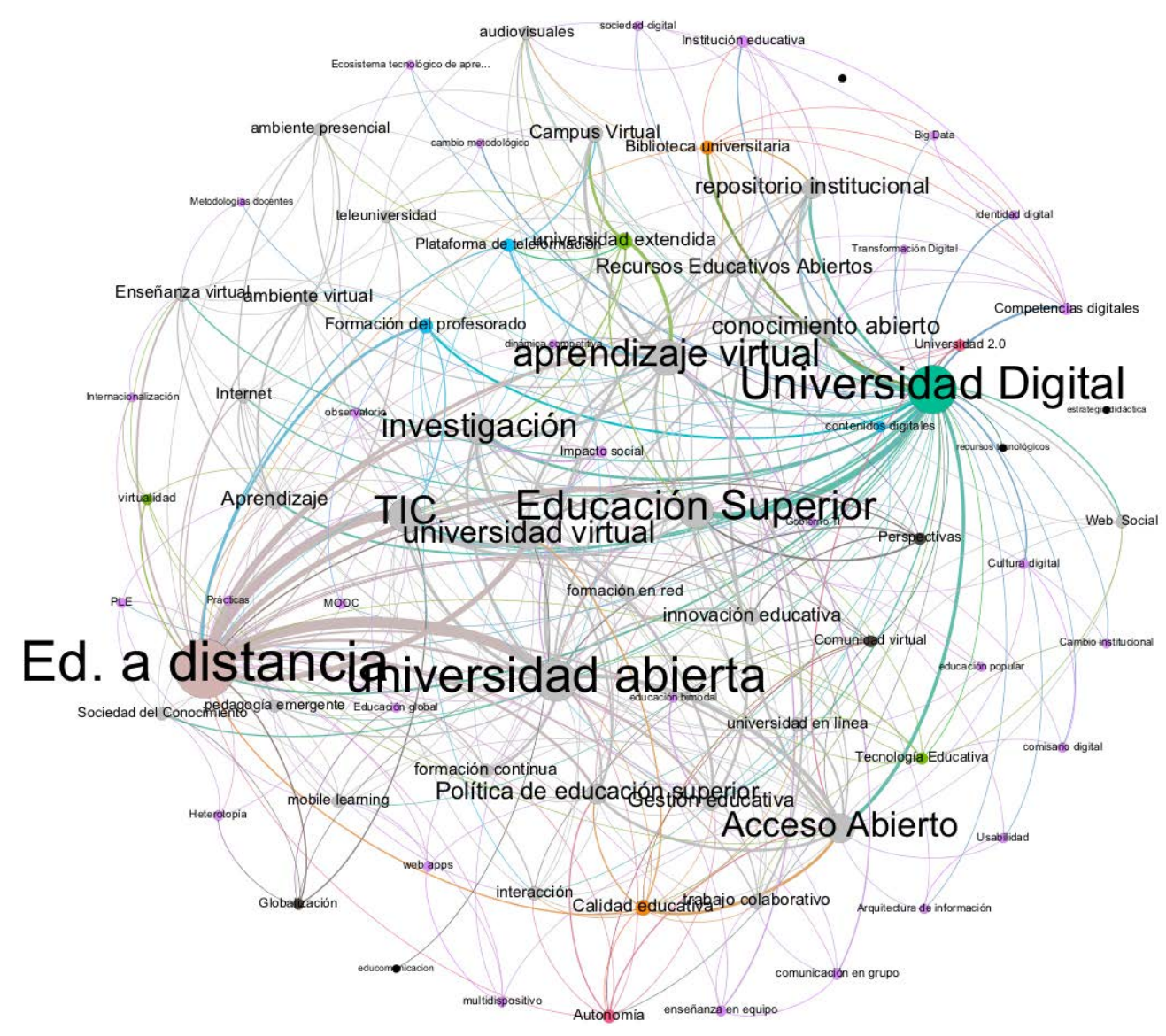

Posteriormente se trabajó con la red de conexiones cruzadas que representa el mapa de la definición de universidad digital a partir de las relaciones entre nuestras palabras clave de búsqueda y las que definen los distintos trabajos encontrados.

De igual modo que en el caso anterior, se realizó el análisis de la distribución siguiendo el mismo procedimiento. Además, se aplicó la herramienta de modularidad para identificar agrupaciones de significado (Grandjean, 2015). Así, se identificaron seis clusters, cuatro de mayor grado (universidad digital, universidad virtual, universidad a distancia y universidad abierta) y dos menores (universidad extendida y universidad en línea).

El grafo resultante (figura 2) describe los diferentes clusters asociados a las palabras clave de partida y un conjunto de conceptos compartidos -que se sitúan en 
el centro. Se identifica claramente un cluster de conceptos alrededor de 'universidad digital' de mayor número de elementos y otros tres que están formados alrededor de 'educación a distancia' (con mayor peso), de universidad virtual y de universidad abierta.

Estos tres clusters entendemos que aportan elementos al significado general de universidad digital, al mismo tiempo que comparten con el cluster de dicho concepto descriptores significativos del campo influyendo en su construcción: 'educación superior', 'investigación', 'interacción', 'acceso abierto', 'aprendizaje', 'aprendizaje virtual', 'enseñanza virtual', 'TIC', 'recursos educativos abiertos', 'repositorio institucional', 'conocimiento abierto', 'universidad 2.0', 'política de educación superior', 'gestión educativa', y que vienen a coincidir con aquellos que más conexiones presentaban en el caso anterior.

Figura 2. Clústeres identificados a partir de la red de conexiones cruzadas entre las palabras de búsqueda y las de los documentos seleccionados

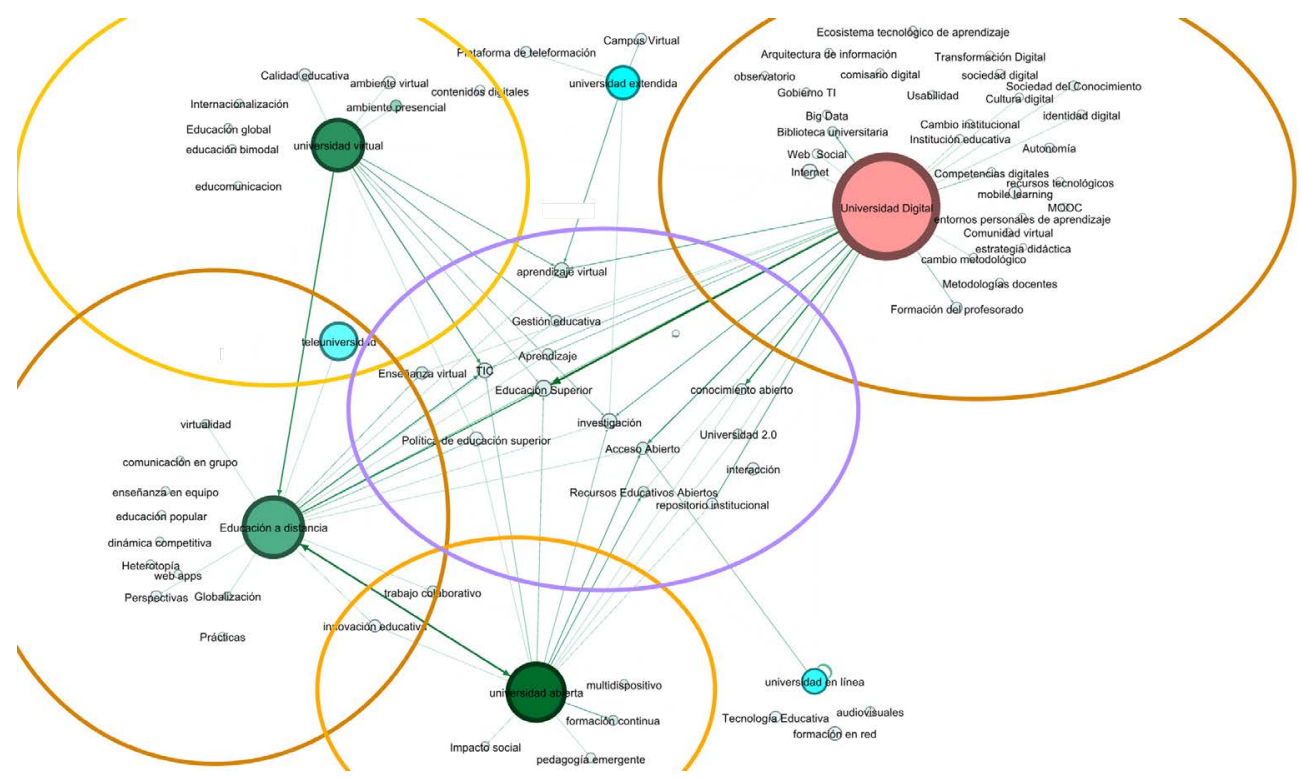

Si circunscribimos la búsqueda a la palabra clave 'universidad digital', encontramos 30 trabajos que vienen definidos por 52 palabras clave y que generan 101 conexiones. Se realizó nuevamente un análisis de la distribución, obteniendo el mapa de la red relacional organizada en tres niveles de importancia de las palabras clave en cuanto a su peso (figura 3). 
Figura 3. Mapa de red de la palabra clave "universidad digital” y términos identificados en los documentos seleccionados

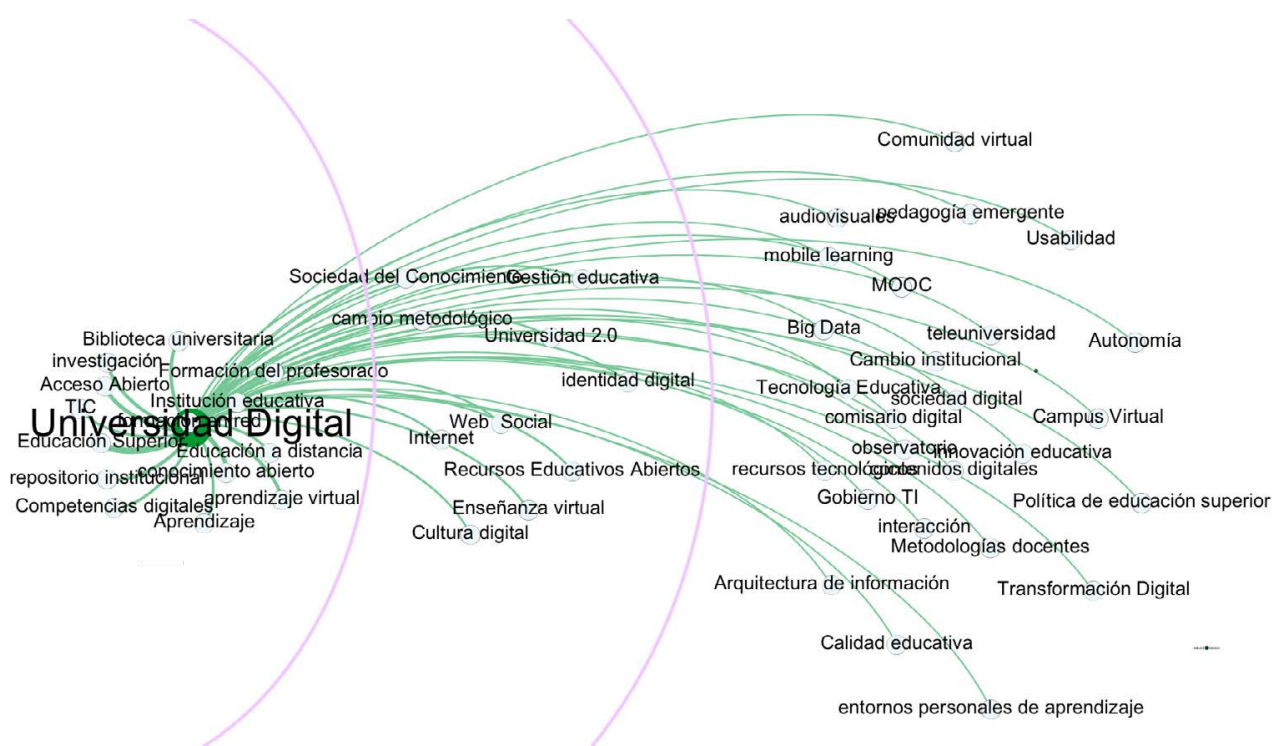

\section{El resumen del procedimiento que se ha seguido puede verse en la figura 4.}

Figura 4. Esquema del procedimiento del análisis semántico seguido en el estudio
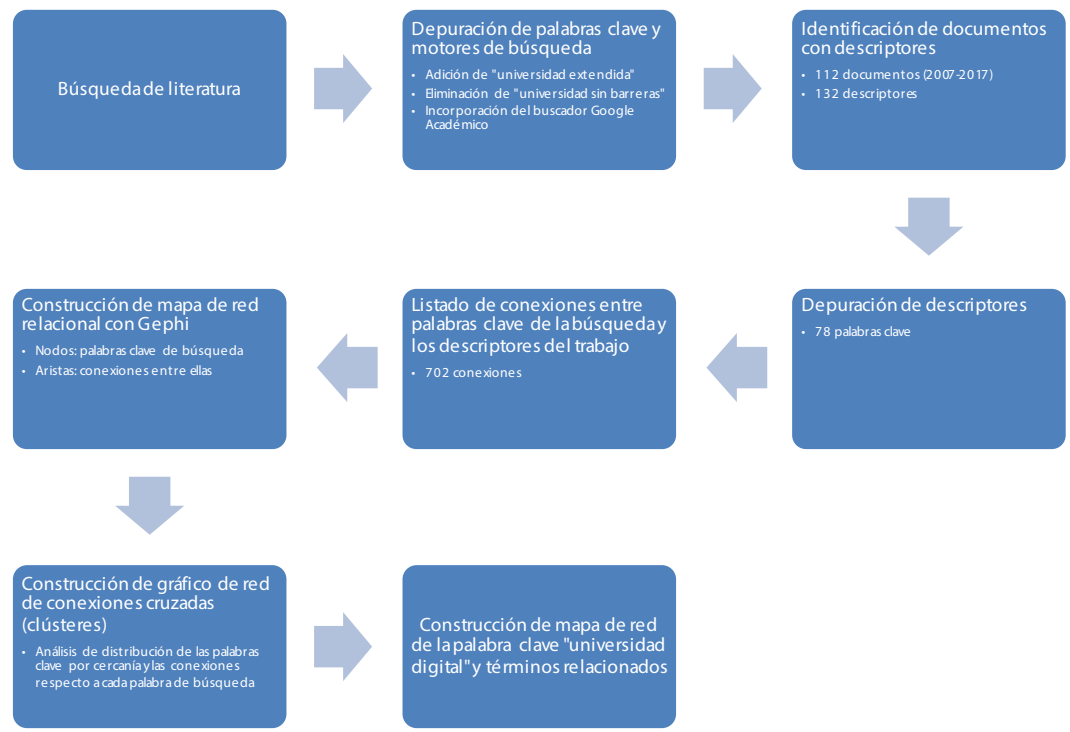


\section{Revisión sistemática de literatura}

En esta fase, se siguieron las indicaciones de Creswell (1994), que indica que la meta de dicha tarea es resumir el estado de conocimientos existentes alrededor de un tema de interés y resaltar los aspectos importantes que quedan por resolver, discutiendo las limitaciones de la investigación actual e indicando posibles direcciones de estudios futuros respecto al concepto de 'universidad digital'.

Siguiendo a Lincoln y Guba (1985), no se determinó con anterioridad al análisis el sistema de codificación, sino que surgió de forma inductiva a partir de la información procedente de las unidades de análisis. La unidad básica de análisis es el resumen o el apartado de introducción de cada uno de los documentos seleccionados previamente en las hojas Excel. En el caso de no encontrar ninguno de ellos, no se tomó en cuenta el documento para la revisión.

El método propuesto por los anteriores autores sigue las siguientes fases: 1) examinar cada resumen/introducción de documento por separado, 2) crear varias categorías, 3) comparar categorías, y 4) lograr la saturación de categorías (repetición de temas).

Dicho análisis y creación de temas se llevó a cabo mediante el software de análisis cualitativo MAXQDA 12, agrupando en temas y categorías con significados similares, de acuerdo a las diferentes secciones de las unidades de análisis.

A partir de la revisión se han identificado las categorías y temas para el concepto de "universidad digital" que se muestran en la tabla 3.

Tabla 3. Categorías y temas identificados en la revisión de la literatura

\begin{tabular}{|l|ll|}
\hline \multicolumn{1}{|c|}{ Categorías } & \multicolumn{1}{c|}{ Temas } \\
\hline Un nuevo modelo de universidad de & - & Nuevas metodologías docentes \\
acuerdo con el Espacio Europeo de & - & Nuevos espacios formativos \\
Educación Superior (EEES) & - & Formación integral e interdisciplinar \\
& - & Formación del profesorado \\
\hline La promoción de una cultura digital & - & Acceso abierto \\
& - & Cultura de innovación \\
\hline & - & El desarrollo de competencias digitales \\
El Gobierno Abierto de la universidad & - & Software y licencias libres \\
\hline La administración electrónica en las & - & Cambios en las políticas institucionales \\
universidades & - & Responsabilidad Social Universitaria \\
\hline $\begin{array}{l}\text { El nuevo rol de las bibliotecas } \\
\text { universitarias }\end{array}$ & - & Ley de Acceso Electrónico de los \\
\hline & - & Ley de Contratación Pública \\
\hline
\end{tabular}




\begin{tabular}{|c|c|}
\hline Categorias & Temas \\
\hline La virtualización de la universidad & $\begin{array}{ll}\text { - } & \text { Campus virtuales } \\
\text { - } & \text { Mobile learning } \\
\text { - } & \text { Webs y portales institucionales } \\
\text { - } & \text { Formación en red y docencia } 2.0 \\
- & \text { Aprendizaje colaborativo } \\
- & \text { Calidad de los programas virtuales }\end{array}$ \\
\hline $\begin{array}{l}\text { El uso y publicación de recursos } \\
\text { educativos abiertos }\end{array}$ & $\begin{array}{ll} & \text { OpenCourseWare } \\
\text { - } & \text { Repositorios institucionales } \\
\text { - } & \text { MOOCs }\end{array}$ \\
\hline La Investigación Abierta & - Acceso y publicación en abierto \\
\hline $\begin{array}{l}\text { La educación permanente o a lo largo de } \\
\text { la vida }\end{array}$ & $\begin{array}{ll}\text { - } & \text { Educación expandida (integración de } \\
\text { ámbitos informales) } \\
\text { - } & \text { Educación de las personas adultas mayores } \\
\end{array}$ \\
\hline
\end{tabular}

\section{RESULTADOS}

La revisión sistemática de la literatura identifica temas similares a los descritos en una exploración inicial del concepto presentada en apartados anteriores.

Retomando la propuesta de Piattini y Mengual (2008), en que se organiza el modelo de universidad en diferentes capas, describimos y discutimos, a continuación, la integración de las categorías y temas que han emergido de la literatura.

Al describir el modelo, dichos autores indican que

las infraestructuras (físicas, lógicas y los servicios de soporte), la capa de gestión TSI y las aplicaciones corporativas de la universidad, todos ellos soportan los diferentes servicios de negocio que, a través de diversas interfaces y canales, y todo ello enmarcado en la estrategia general de la Universidad y en las políticas de gobierno de las TIC, llegarán a todos los miembros de la comunidad universitaria y a la sociedad en general (Piattini y Mengual, 2008, p. 21).

En los niveles de infraestructuras físicas y lógicas no hay nada específico descrito en la literatura revisada, quizá por su excesiva concretización tecnológica.

El tercer nivel identificado, en el modelo de Piattini y Mengual (2008), es el que nos es de utilidad para ubicar lo que entienden los diferentes autores iberoamericanos por el concepto de 'universidad digital', que no es otro que el referido a la capa de servicios (ver figura 5), entre los que destacan:

- La gestión tecnológica. Aquí tendría cabida el tema vinculado al nuevo rol del jefe de tecnologías identificado en la categoría sobre el gobierno abierto de la universidad (Llorens, 2017).

- Los sistemas de gestión de la universidad, donde se puede ubicar todo el apartado vinculado a la administración electrónica, que contempla diversas leyes que buscan implementar estrategias de digitalización de las universidades, como la 
Ley de Acceso Electrónico de los Ciudadanos a los Servicios Públicos y la Ley de Contratación Pública (García-Peñalvo, 2011). En este mismo apartado, también tendría cabida la categoría vinculada con el gobierno abierto, el camino hacia la transparencia de procesos, la democratización de la educación superior y la autonomía universitaria (Esteve, Mottareale y Parejo, 2011; Pascual y Teruel, 2013; Sánchez González, Paniagua, Mora y Rancaño, 2014; Solís, 2014). Como temas, cabría destacar la importancia de la responsabilidad social por parte de las universidades y los cambios institucionales.

- Los canales, que se refieren a las herramientas específicas a partir de las cuales se hace efectivo el nuevo modelo, como la TV digital o el correo electrónico. No se identifican referencias específicas a este aspecto en la literatura, pues se trata de algo muy concreto -ya de operacionalización- para las concepciones de la universidad digital.

- Los interfaces apuntan a los diferentes portales que conectan los canales y los diferentes servicios de negocio dela universidad. Por tanto, se incluyen los portales web, el campus virtual y otros portales varios. Este es un tema que aparece en el apartado de la virtualización de la universidad y que está vinculado a la presencia en la red de la universidad a través de los portales web institucionales (Ortega y Montero, 2013), y a la docencia online mediante los campus virtuales (Llorens, 2014; Marquès, 2008; Pérez Lorido, 2007).

- El conjunto de todos los servicios de negocio podría equivaler a la concepción frecuente de la universidad digital como un nuevo modelo de universidad que sigue las directrices del EEES y que se centra, especialmente, en un mayor rol proactivo y aprendizaje autónomo de los estudiantes, el desarrollo de nuevas metodologías docentes y nuevos espacios formativos, la formación del profesorado en este nuevo modelo y la formación integral e interdisciplinar de los estudiantes (Gros, 2015; Trujillo, 2010). Algunos de los referidos en la literatura se describen a continuación:

- Docencia/eLearning, que también se ha mencionado en la capa de interfaces, pero aquí además incluiría los procesos de comunicación, enseñanza y aprendizaje en este contexto de docencia online o formación en red, incluyendo el aprendizaje colaborativo en entornos y herramientas virtuales (Lupion y Marques, 2012). Los temas vinculados con la virtualización de la universidad a nivel educativo son los más frecuentes mencionados en la literatura. También fueron explorados previa y parcialmente desde la concepción de universidad digital como enfoque estratégico o unidad académica.

- Investigación y la gestión de conocimiento, donde hablaríamos de la investigación abierta a través del acceso y publicación en abierto (Contreras, 2016), del uso y publicación de recursos educativos abiertos en forma de objetivos educativos, OpenCourseWare, MOOCs y repositorios institucionales (López Medina y Zorita, 2009), del nuevo rol de las bibliotecas universitarias, 
desde la integración bibliotecaria y la consideración de nuevas técnicas desde las Humanidades Digitales y el Big Data (Llorens, 2011; Martínez Cardama y Caridad, 2016), o de la universidad digital como integradora y promotora de una cultura digital (universidad digital como práctica), en la que se promueva el acceso abierto, una cultura de innovación, el uso de software y licencias libres y el desarrollo de competencias digitales (Freire y Schuch, 2010), todos ellos temas recurrentes conectados con el significado de universidad digital y, parcialmente, con la concepción analizada previamente en torno a la presencia de las universidades en las redes.

- Cabe destacar el servicio de cuadro de mando y calidad, que integraría la preocupación por la calidad de los programas educativos virtuales (ArayaCastillo, 2015; Orozco, 2014).

Figura 5. Modelo de universidad digital integrando la capa de servicios propuesta por

Piattini y Mengual (2008) y las categorías y conceptos identificados en el estudio

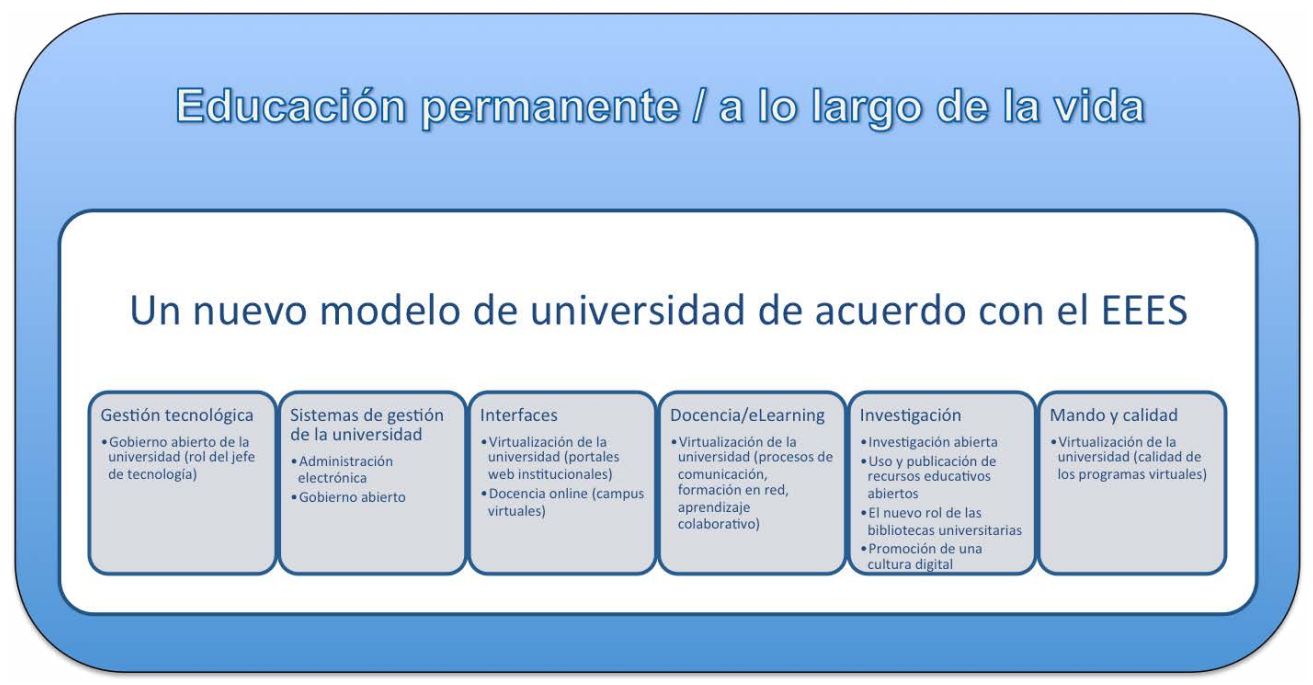

Una concepción más amplia de universidad digital apunta a la educación permanente o a lo largo de la vida, incluyendo la educación expandida -integrando otras formas de aprendizaje más allá de la educación formal- (Freire y Schuch, 2010) y la educación de las personas adultas mayores (de Cássia y Andressa, 2015), que no se encontraría representada en el modelo de universidad digital presentado por Piattini y Mengual (2008). 


\section{DISCUSIÓN Y CONCLUSIÓN}

$\mathrm{Al}$ analizar los resultados de la revisión de trabajos, se distingue cierta diferencia en el enfoque entre la literatura publicada en España y en América Latina. Mientras, por una parte, la perspectiva de España se centra en el nuevo modelo de Universidad basado en el EEES y la promoción de la cultura digital, seguidos por temas mencionados con menor frecuencia, como el rol de las bibliotecas, el gobierno abierto y la educación permanente; por la otra, la de América Latina pone mayor énfasis en la digitalización de la universidad, el uso de campus virtuales y la calidad de la educación virtual.

Como limitaciones del concepto, se identifican claramente la falta de consideración de asuntos, tales como la de inversión en infraestructura electrónica y de calidad de la formación docente, así como la evasión en los cursos de educación a distancia (Bittencourt y Mercado, 2014) y la pobre relación entre empresa y universidad (Contreras, 2016), en el ámbito latinoamericano; y la introducción de procedimientos de calidad como posibles obstáculos a las oportunidades de innovación (Freire y Schuch, 2010), en España.

A partir de los hallazgos descritos y como conclusión, puede decirse que el concepto de universidad digital sigue siendo un concepto abierto y polisémico, que se construye sobre una serie de conceptos clave en la reflexión actual y en los estudios de prospectiva sobre la universidad. Esto puede verse, tanto a nivel de España y América latina (Fernández y Llorens, 2013), como a nivel internacional (Siemens, Gasevic y Dawson, 2015; Jones, 2013). Principalmente puede conectarse con 'educación superior', 'universidad abierta', 'universidad virtual', 'aprendizaje virtual', 'investigación', 'acceso abierto'.

A juzgar por la revisión de la literatura, la universidad digital es, al mismo tiempo, un modelo de universidad con gobierno abierto y la administración electrónica y la virtualización de la universidad. Así, hace referencia a:

- La dotación de infraestructuras tecnológicas para la universidad o la implementación de políticas institucionales de digitalización de la universidad.

- Un modelo de universidad con gobierno abierto que tiende hacia la incorporación de ámbitos de aprendizaje diferentes a la educación formal, que se extiende a lo largo de la vida, y que se basa en estrategias de aprendizaje centradas en el aprendiz, de carácter autorregulado pero también colaborativo, apoyado por el nuevo rol del docente.

- La promoción de una cultura digital embebida en la organización, donde la integración de diferentes actores educativos adquiriendo un nuevo rol, como las bibliotecas universitarias, es fundamental para el apoyo de la investigación abierta, o el uso y la publicación de recursos educativos y repositorios institucionales abiertos. 
- La administración electrónica y la virtualización de la universidad en forma de webs y portales institucionales, formación en red a través de campus virtuales, como parte del desarrollo natural de la sociedad del conocimiento en la que vivimos.

Para finalizar, puede decirse que el adjetivo digital sobra (o al menos, sobrará), dado que, en la actualidad, ya todo es digital. El entorno digital se ha ido convirtiendo en una nueva normalidad, generando un contexto que altera las reglas: lo transforma todo, lo hace a una velocidad exponencial y cambia el factor estratégico de desarrollo. Por lo tanto, las instituciones necesitan activar la capacidad de cambio y será mejor cuanto antes se identifique el concepto de universidad con el de universidad digital. Esto supone un cambio de modelo y, para que se dé este cambio, tiene que haber una visión hacia la digitalización, hacia la transformación digital. Esta visión viene marcada por dos parámetros: la intensidad digital (cómo se digitalizan los procesos y que podemos analizar mediante, p.e., los indicadores que proponen en UNIVERSITIC) y la intensidad de la capacidad transformadora (una cultura y un liderazgo positivo hacia el mundo digital).

\section{NOTAS}

1. Universidad de Granada: https://udigital.ugr.es/?login form register

2. OCU: http://www.ocu.es/productos/universitas-xxi-universidad-digital/

3. Universidad de la Serena: http://universidad.userena.digital

\section{REFERENCIAS BIBLIOGRÁFICAS}

Aguado-López, E., Rogel-Salazar, R., Becerril-García, A., y Baca-Zapata, G. (2009). Presencia de universidades en la Red: la brecha digital entre Estados Unidos y el resto del mundo. RUSC. Revista de Universidad y Sociedad del Conocimiento, 6(1), 1-17. doi: 10.7238/ rusc.v6i1.18.

Araya-Castillo, L. (2015). Dinámica competitiva de las universidades en Chile y la necesidad de potenciar el mercado de la educación a distancia. Aposta. Revista de Ciencias Sociales, 64, 1-30. Recuperado de http://www.redalyc.org/ articulo.0a?id=495950260007

Bastian, M., Heymann, S., y Jacomy, M. (2009). Gephi: An open source software for exploring and manipulating networks. In Proceedings of the Third International ICWSM Conference (pp. 361-362). Menlo Park, CA: AAAI Press. Recuperado de http://www.aaai.org ocs/index.php/ICWSM/og/paper/ viewFile/154Forum/1009m

Bittencourt, I. M., y Mercado, L. P. L. (2014). Evasão nos cursos na modalidade de educação a distância: estudo de caso do Curso Piloto de Administração da UFAL/UAB. Ensaio: Avaliação e Políticas Públicas em Educação, 22(83), 465-504. doi: 10.1590/So10440362014000200009.

Burman, J. T., Green, C. D., y Shanker, S. (2015). On the Meanings of SelfRegulation: Digital Humanities in Service 
of Conceptual Clarity. Child Development, 86(5), 1507-1521. doi: 10.1111/cdev.12395. de Cássia, R., y Andressa, P. (2015). Universidad abierta a las personas adultas mayores: espacio de pedagogía social, educación y empoderamiento. Actualidades Investigativas en Educación, 15(3), 1-18. doi:10.15517/aie. v15i3.20672.

Contreras, R. (2016). La responsabilidad de las Universidades en la e-Investigación. En R. Roig (Coord.), Tecnología, innovación e investigación en los procesos de enseñanza-aprendizaje (2027-2033). Barcelona: Octaedro. Recuperado de http://rua.ua.es/dspace/ handle/10045/61787

Creswell, J. (1994). Research design qualitative and quantitative approaches. Thousand Oaks: Sage Publications.

Esteve, F. M., Mottareale, D., y Parejo, J. L. (2011). La implementación estratégica de las TIC en la Universidad: competencia digital y Open Government. Congreso Internacional "Educación Mediática y Competencia Digital". Seqovia. Recuperado de http://www. educacionmediatica.es/comunicaciones/ Eje\%202/Francesc\%20Marc\%20 Esteve,\%20Daria\%20Mottareale,\%20 Jos\%C3\%A9\%20Luis\%20Parejo.pdf

Fernández, A., y Llorens, F. (Coord.) (2013). UNIVERSITIC Latinoamérica 2013: Descripción y Gestión de las Tecnologías de la Información en las universidades latinoamericanas. Organización Universitaria Interamericana (OUIIOHE), Montreal (CA).

Freire, J., y Schuch, K. (2010). Políticas y prácticas para la construcción de una Universidad Digital. La Cuestión Universitaria, 6, 85-94. Recuperado de: http://polired.upm.es/index.php/ lacuestionuniversitaria/article/view/3401

García-Peñalvo, F. (2011). La Universidad de la próxima década: La Universidad Digital. En C. Suárez Guerrero y F. García Peñalvo (Coord.), Universidad y
Desarrollo Social en la Web (181-197). Washington DC, USA: Editandum.

Gómez, J. (Ed.) (2016). UNIVERSITIC 2016. Análisis de las TIC en las Universidades Españolas. Madrid: Crue Universidades Españolas. Recuperado de: http://www. crue.org/Publicaciones/Documents/ Universitic/2016.pdf

Grandjean, M. (2015). GEPHI - Introduction to network analysis and visualization. Recuperado de http://www. martingrandjean.ch/gephi-introduction

Green, C. D., Feinerer, I., y Burman, J. T. (2015). Searching for the structure of early American psychology: Networking Psychological Review, 1894-1908. History of Psychology, 18, 15-31. doi: 10.1037/aoo38406.

Gros, B. (2015). La caída de los muros del conocimiento en la sociedad digital y las pedagogías emergentes. Education in the knowledge society, 16(1), 58-68. doi: 10.14201/eks20151615868.

Jones, C. (2013). The digital university: a concept in need of definition. En R. Goodfellow y M. R. Lea (Eds.), Literacy in the Digital University. Critical perspectives on learning, scholarship, and technology (162-172). Routledge, London.

Kampylis, P., Punie, Y., y Devine, J. (2015). Promoting Effective Digital-Age Learning - A European Framework for Digitally-Competent Educational Organisations. EUR 27599 EN. doi: 10.2791/54070.

Laviña Ortueta, J., y Mengual Pavón, L. (Coord.) (2008). Libro Blanco de la Universidad Digital 2010. Barcelona: Ariel. Recuperado de https://www. fundaciontelefonica.com/arte cultura/ publicaciones-listado/pagina-itempublicaciones/itempubli/22

Lara, T. (2009). El papel de la Universidad en la construcción de su identidad digital. Revista de Universidad y Sociedad del Conocimiento (RUSC), 6(1), 15-21. 
Recuperado de http://www.redalyc.org/ articulo.oa?id=78011179009

Larenas, M. (2017). Presentación. En ACTAS TICAL 2017 San José, Costa Rica.

Leiva-Aguilera, J. (2016). Transformación digital y biblioteca universitaria. Anuario ThinkEPI, 10, 79-82. doi: 10.3145/ thinkepi.2016.12.

Lincoln, Y. S., y Guba, E. G. (1985). Naturalistic inquiry. Beverly Hills, CA: Sage Publications.

Llorens, F. (2011). La biblioteca universitaria como difusor de la innovación educativa. Estrategia y política institucional de la Universidad de Alicante. ARBOR Ciencia, Pensamiento y Cultura, 187(3), 89-100. doi: 10.3989/arbor.2011.Extra-3n3132.

Llorens, F. (2014). Campus virtuales: de gestores de contenidos a gestores de metodologías. Revista de Educación a Distancia, 42, 1-12. Recuperado de http:// revistas.um.es/red/article/view/236561

Llorens, F. (2017). El papel del CIO en la Universidad. Conferencia Cátedra Santander-UA de Transformación Digital (pp.1-5). Recuperado de http://rua.ua.es/ dspace/handle/10045/63136

Llorens, F. (Coord.) (2012). Tendencias TIC para el apoyo a la Docencia Universitaria. Madrid: CRUE. Recuperado de http://www.crue.org Publicaciones/Documents/Universitic/ Tendencias TIC 2012.pdf

López Medina, A. y Zorita, L. (2009). "e-spacioUNED", el repositorio institucional de una Universidad a Distancia. II Jornada Documat: digitalizaciones $y$ documentación científica en matemáticas. Madrid. Recuperado de http://eprints.ucm. es/9751

Lupion, P., y Marques, L. M. (2012). Educação virtual nas universidades: as contribuições da aprendizagem colaborativa. Rev.hist.educ.latinoam, 14(19), 175-204. Recuperado de http://www.scielo.org.co/scielo. php? script $=$ sci abstract\&pid $=$ So122 72382012000200009\&lng=pt\&nrm=is

Marquès, P. (2008). Impacto de las TIC en la enseñanza universitaria. DIM: Didáctica, Innovación y Multimedia, 11. Recuperado dewww.raco.cat/index.php/DIM/article/ view/87133

Martínez Cardama, S., y Caridad, M. (2016). El bibliotecario académico en la Universidad Digital: nuevos roles profesionales. In Informação e inovação para a pesquisa e o desenvolvimento social (pp. 303-318). Brasil.

Martín-González, M. (Ed.) (2016). Buenas Prácticas sobre la Universidad Digital. Madrid: Cátedra UNESCO de Gestión y Política Universitaria. Recuperado de: http://www.catedraunesco.es/13noticias/actualidad/374-buenaspr\%C3\%A1cticas-sobre-la-universidaddigital.htm

Musse, J. I. (2015). Oportunidades para las universidades de América Latina. En E. Chinkes (Coord.), Las Tecnologías de la Información y la Comunicación. Potenciando la Universidad del Siglo XXI (49-53). TICAL, RedClara, Buenos Aires.

Orozco, L. M. (2014). Estudio comparativo de los modelos de evaluación de la calidad del e-learning en el Sistema de Universidad Virtual de la Universidad de Guadalajara -México y propuesta complementaria. Universitat de Lleida. (Tesis Doctoral). Recuperado de http://www.tesisenred. net/handle/10803/285341

Ortega, S., y Montero, Y. H. (2013). Análisis y evaluación de sitios web universitarios españoles a partir del proceso de Bolonia. Perspectivas em Ciência da Informação, 18(4), 70-92. doi. 10.1590/S141399362013000400006.

Pascual, J., y Teruel, E. (2013). Universidad Abierta: reutilización de la información universitaria. RUIDERAe: Revista de Unidades de Información, 4, 1-22. Recuperado de https://revista.uclm.es index.php/ruiderae/article/view/398 
Pérez Lorido, M. (2007). Asignaturas virtuales en universidades presenciales: perspectivas y problemas. Pixel-Bit. Revista de Medios y Educación, 30, 7384. Recuperado de https://idus.us.es/ xmlui/handle/11441/45639

Piattini, M., y Mengual, L. (2008). Universidad digital 2010. En J. Laviña Ortueta y L. Mengual Pavón (Coord.), Libro Blanco de la Universidad Digital 2010 (5-27). Barcelona: Ariel.

Píriz, S., (Dir.). (2013). UNIVERSITIC 2013: Descripción, Gestión y Gobierno de las TI en el SUE. CRUE, Madrid. Recuperado de http://www.crue.org/Publicaciones/ Documents/Universitic/2013.pdf

Price, R. (2017). Las redes sociales como plataformas de aprendizaje. En R. Chinkes (Coord.), Potenciando la Universidad del Siglo XXI. Soluciones TIC para pensar la universidad del futuro (67-75). TICAL, RedCLARA, Buenos Aires.

Ribeiro, L., de Castro, P., y Mennielli, M. (2016). Final Report. EUNIS - EUROCRIS Joint Survey on CRIS and IR. ERAI Report.

Sánchez González, M., Paniagua, F. J., Mora, L., y Racano, L. (2014). Innovación y Open Government como claves para una universidad abierta y participativa. Estrategias y resultados en la UNIA. Telos: Cuadernos de comunicación e innovación, 97, 93-102. Recuperado de https://telos.fundaciontelefonica.com url-direct/pdf-generator?tipoContenido= articuloTelos\&idContenido=2014042309 530003\&idioma $=\mathrm{es}$
Siemens, D., Gasevic, D., y Dawson, S. (2015). Preparing for the Digital University: a review of the history and current state of distance, blended, and online learning. Athabasca University, University of Edinburgh, University of Texas Arlington, University of South Australia.

Solís, F. M. (2014). ¿Y las Universidades qué? Universidad Abierta: algo más que Gobierno Abierto y Open Data. Actualidad administrativa, 7-8, 841-849. Recuperado de https://riunet.upv.es/ handle/10251/52285

TICAL (2016). Actas TICAL 2016 Sexta Conferencia de Directores de Tecnología de Información, TICAL2016 Gestión de las TICs para la Investigación y la Colaboración. Buenos Aires, Argentina.

Trujillo, J. M. (2010). Universidad digital 2010. Nuevas competencias metodológicas, estratégicas y alfabetización informacional para el presente y el futuro europeo. Multiárea: revista de didáctica, 5, 339-364. Recuperado de https: / / www.researchgate.net publication/288466688 Universidad Digital 2010 Nuevas competencias metodologicas estrategias y alfabetizacion informacional para el presente y futuro europeo

Uceda, J. (Dir.) (2011). UNIVERSITIC 2011: Descripción, gestión y gobierno de las de las TI en el SUE. CRUE, Madrid. Recuperado de http://www. crue.org/Publicaciones/Documents/ Universitic/2011.pdf

\section{PERFIL ACADÉMICO Y PROFESIONAL DE LOS AUTORES}

Jesús Salinas Ibáñez. Investigador principal del Grupo de Tecnología Educativa de la Universitat de les Illes Balears (UIB, España). Doctor en Filosofía y Ciencias de la Educación, Licenciado en Filosofía y Letras (Sec. CC Educación) y Diplomado en Profesorado de EGB. Docente de Grado, Postgrado y Doctorado. Director del Institut de Recerca i Innovació Educativa de la UIB. Líneas de trabajo: e-learning, educación flexible, itinerarios de aprendizaje flexible con mapas 
conceptuales, aprendizaje autorregulado, diseño del e-learning, entornos personales de aprendizaje.

E-mail: jesus.salinas@uib.es

\section{DIRECCIÓN DEL AUTOR}

Institut de Recerca i Innovació Educativa (IRIE).

Facultad de Educación

Universidad de las Islas Baleares

Ed. Sa Riera

C/ de Miquel dels Sants Oliver, 2

Palma (España)

Victoria I. Marín Juarros. Investigadora postdoctoral en la Universidad de Oldenburg (Alemania). Colaboradora de investigación del IRIE con el Grupo de Tecnología Educativa de la Universitat de les Illes Balears (UIB, España). Doctora en Tecnología Educativa por la UIB. Anteriormente profesora asociada del área de Tecnología Educativa en el Departamento de Pedagogía Aplicada y Psicología de la Educación de la UIB. Líneas de trabajo: entornos personales de aprendizaje (PLE) en la educación superior, las redes personales de aprendizaje para el desarrollo profesional, ePortfolios, formación inicial digital docente, el uso de herramientas web 2.o y redes sociales para la formación, curación de contenidos para la actualización docente.

E-mail: victoria.marin@uni-oldenburg.de

\section{DIRECCIÓN DEL AUTOR}

Faculty of Education and Social Sciences - Institut für Pädagogik

Carl von Ossietzky Universität Oldenburg

Ammerländer Heerstraße 138

Oldenburg (Alemania)

Fecha de recepción del artículo: 14/12/2017

Fecha de aceptación del artículo: 19/01/2018

\section{Como citar este artículo:}

Salinas Ibáñez, J. y Marin, V. I. (2018). Las diferentes concepciones de la universidad digital en Iberoamérica. RIED. Revista Iberoamericana de Educación a Distancia, 21(2), pp. 97-118. doi: http://dx.doi.org/10.5944/ried.21.2.20653 